\title{
Protocol for Generating Cell Lines Specific to Patients with Duchenne Muscular Dystrophy and Able to Induce Pluripotent Stem Cells
}

\author{
Dominique Mornet* \\ University of Montpellier Phymedexp, Inserm, CNRS, France
}

*Corresponding author: Dominique Mornet, previously DR2CNRS, Phymedexp, University of Montpellier Phymedexp,

Inserm, CNRS, Montpellier France

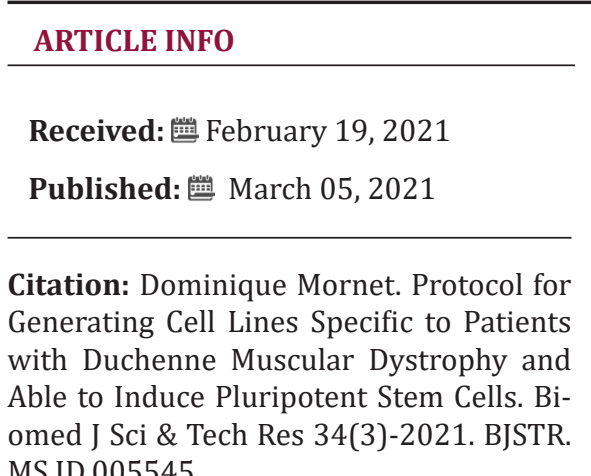

ABSTRACT

Abbreviations: HPSC: Human Pluripotent Stem Cells; ROS: Reactive Oxygen Species; NOS: Nitric Oxide Synthase; CC: Cardiac Cells; EBs: Embryoid Bodies

\section{Short Communication}

Pluripotent stem cells derived from DMD heart cells reflect human pathophysiology in vitro. The literature indicates the existence of numerous independent lines of human pluripotent stem cells deficient in dystrophin (hPSC) derived from patients with DMD with a mutation, in the DMD gene, generated by the CRISPR / Cas9 technique. In this context, it is created a type of stem cell called DMDhPSC and derived from heart cells (CC) to provide a new original specific model of human DMD-CC disease. It is thus, observed that cells carrying mutations were less inclined to differentiate into CC. DMD-CC cells have an improved rate of cell death over time. In addition, alteration of ion channel expression in terms of potassium (overexpression of Kir2.1) and manipulation of calcium (overexpression of dihydropyridine receptors) are demonstrated. DMD-CC showed an increased duration for transient calcium increase compared to the control of the same age, suggesting poor management of calcium release. The results of the mechanical disorders observed are as follows: hypo contractility, bradycardia, variability in the increase in heart rate and a blunt b-adrenergic response linked to the remodeling of the expression of b-adrenergic receptors in DMD- cells. CC. Overall, these results indicate that DMD-CC model cells are functionally associated to dystrophin deficiency. These cells are able to recapitulate the functional defects and cardiac melting observed in cardiomyopathy.

This cell model offers a valuable tool for studying the progression of human cardiomyopathy and monitoring the benefit of in vitro therapies. This short notice focus light on a research team that has already demonstrated its expertise in reprogramming fibroblasts from two independent DMD patients with the complete loss of dystrophin expression, because they carry deletions of exons 4550 (DMD02, MUNIi001-A) and 48- 50 (DMD03, MUNIi003-A). The resulting human stem cells called "hiPSC" possess the expression of pluripotency markers (NANOG, OCT4, SSEA4), a strong capacity for differentiation in the three germ layers, a normal karyotype, a genetic identity with the fibroblasts of parental origin not expressing dystrophin. These stem cells are dshown as forming new cell lines [1]. In addition, this same team had recently established, by reprogramming human pluripotent stem cell lines deficient in dystrophin (hiPSC), that this induced the release of reactive oxygen 
species (ROS) following a deregulated activity of the three isoforms of nitric oxide synthase (NOS). The release of ROS, induced by NOS, leads to DNA damage and genomic instability of these stem cells [2]. Thus, this type of stem cell called DMDhPSC derived from cardiac cells (CC) and exhibiting a dystrophin deficiency also has a lower efficiency for differentiation in CC but reveals a similar relative expression of cardiac markers with a tendency to increase the expression of Connexin 43 [3].

In conclusion, the dystrophin-deficient DMD-CC model cells are a cellular model capable of recapitulating the functional defects and cardiac wasting observed in this X-linked cardiomyopathy and may help to better define cardiac abnormalities in DMD patients. It offers a precise tool for studying the progression of this disease in humans and is useful for testing future therapeutic strategies. According to such expertise, this research team describe in a recent publication [4], how to generate iPSC lines from three different DMD patients carrying respectively deletions of exons 1, 52 and 55 in the dystrophin gene. Such reprogrammed iPSC lines showed their pluripotency to differentiate into the three germ layers by the formation of embryoid bodies (EBs). The ability of such DMD iPSC resulting in DMD EBs were able to express specific ectoderm, endoderm and mesoderm markers revealed b RT-qPCR as this is illustrated in their paper and all characteristics of these iPSC lines are given [4]. Notice also that such DMD iPSC, described above, presented no major genomic aberrations after chromosomal microarray that was performed using Sure Print G3 Human CGH Microarray Kit, $8 \times 60 \mathrm{~K}$ with overall median probe $41 \mathrm{~Kb}$ genomewide median probe spacing. The goal of generating iPSC lines from 3 DMD patients to obtain iPSC derived cardiomyocytes (iPSC$\mathrm{CMs}$ ) provide a power tool allowing to decipher the molecular and pathophysiological mechanisms causing aberrant cellular changes in DMD iPSC-CMs and to test drugs to treat DMD-associated cardiac disease.

\section{References}

1. Jelinkova S, Markova L, Pesl M, Valáškova I, Makaturová E, et al. (2019) Generation of two Duchenne muscular dystrophy patient-specific induced pluripotent stem cell lines DMD02 and DMD03 (MUNIi001-A and MUNIi003-A). Stem Cell Res 40: 101562.

2. Jelinkova S, Fojtik P, Kohutova A, Vilotic A, Marková L, et al. (2019) Dystrophin Deficiency Leads to Genomic Instability in Human Pluripotent Stem Cells via NO Synthase-Induced Oxidative Stress. Cells 8(1): 53.

3. Jelinkova S, Vilotic A, Pribyl J, Aimond F, Salykin A, et al. (2020) DMD Pluripotent Stem Cell Derived Cardiac Cells Recapitulate In vitro Human Cardiac Pathophysiology. Front Bioeng Biotechnol 8: 535.

4. Souidi M, Amédro P, Meyer P, Desprat R, Lemaître JM, et al. (2020) Generation of three Duchenne Muscular Dystrophy patient-specific induced pluripotent stem cell lines DMD_YoTaz_PhyMedEXp, DMD RaPer_PhyMedEXp, DMD_OuMen_PhyMedEXp (INSRMi008-A INSRMi009-A and INSRMi010-A). Stem Cell Res 49: 102094.

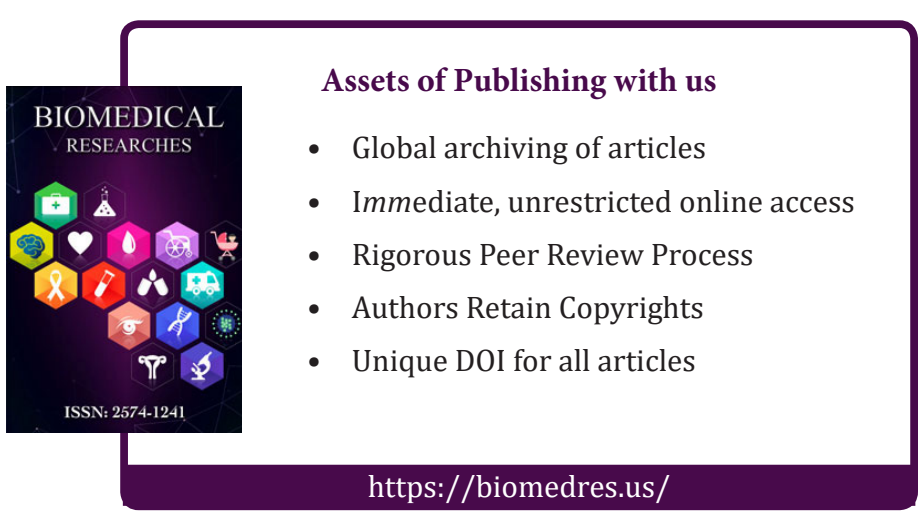

